\title{
Familia y enfermedad crónica pediátrica
}

\section{Family and chronic paediatric illness}

\author{
C. Grau ${ }^{1}$, M. Fernández Hawrylak ${ }^{2}$
}

\section{RESUMEN}

La enfermedad pediátrica es siempre un problema familiar. La hospitalización, los tratamientos y las secuelas constituyen un desafío para la familia. En este trabajo describimos las alteraciones estructurales, procesuales y emocionales que se producen en la dinámica familiar. Consideramos que la intervención del niño enfermo debe hacerse siempre en el contexto familiar y proponemos un modelo de intervención multidimensional centrado en la singularidad de las familias y en sus necesidades, en los apoyos disponibles en su entorno natural, en el desarrollo de las competencias y de la resiliencia y en la organización de servicios centrados en el usuario y coordinados con todos los servicios que ofrece la comunidad.

Palabras clave. Enfermedad crónica pediátrica. Familia. Resiliencia. Orientación familiar.

\begin{abstract}
Pediatric illnesses are always a family problem. Hospitalization, treatments and their long term consequences constitute a challenge for the family. In this paper, we describe the structural, procedural and emotional alterations that affect the family dynamic We argue that the child should be treated within the family context and propose a multi-dimensional intervention model centered on the family's singularities and specific needs, the support available in their environment, the development of capacities and resilience, and also the organization of user-centered services that are coordinated with all the services provided by the community.
\end{abstract}

Key words. Paediatric chronic disease. Family. Resilience. Family counselling.
1. Escuela Universitaria de Magisterio "Ausias March». Universidad de Valencia.

2. Facultad de Humanidades y Educación. Universidad de Burgos.

Recepción el 9 de marzo de 2010

Aceptación provisional el 15 de abril de 2010

Aceptación definitiva el 31 de mayo de 2010

\section{Correspondencia}

Claudia Grau Rubio

Escuela Universitaria de Magisterio «Ausias March»

Departamento de Didáctica y Organización Escolar

Universidad de Valencia

Apartado de correos 22045

46071 Valencia / España

E-mail: claudia.grau@uv.es 


\section{INTRODUCCIÓN}

La enfermedad del niño es siempre un problema familiar y su calidad de vida está íntimamente relacionada con el apoyo familiar que recibe ${ }^{1}$.

Los padres tienen que adaptarse a las características de cada enfermedad en sus diferentes fases (diagnóstico, tratamientos...) restructurando su vida familiar. La enfermedad puede ser impredecible, requiere atención constante y llegar a formar parte de la vida familiar, produciendo cambios estructurales, procesuales y emocionales en la familia.

Ante la enfermedad crónica, los padres reaccionan de distinta manera según su grado de vulnerabilidad o según su grado de resiliencia (fortaleza y competencia) ${ }^{2}$. Sus reacciones dependen de numerosos factores como: experiencia en situaciones de crisis y problemas médicos, estatus socio-económico, nivel de conocimientos, calidad de los servicios sanitarios y educativos y sistemas de apoyo.

En el trabajo y colaboración con las familias los profesionales deben: respetar la singularidad de éstas, basarse en sus necesidades, tener una comprensión multidimensional del problema, desarrollar competencias en los padres y la resiliencia, contar con los sistemas de apoyo del entorno natural y coordinar la acción de los servicios públicos (sanitarios, educativos y de servicio social) y los que ofrece la sociedad civil (ocio, asociaciones, voluntariado...). Se trata de crear un clima de equipo que cambie los sentimientos de impotencia de la familia, de los profesionales y de los miembros de la comunidad $^{3}$.

\section{REPERCUSIONES DE LAS FASES DE LA ENFERMEDAD EN LA FAMILIA}

Las familias con un hijo enfermo tienen que adaptarse a las diferentes fases de la enfermedad.

- Fase de crisis: comprende el periodo previo al diagnóstico. En esta fase se manifiestan los síntomas y existe la sospecha de que algo no va bien. En este periodo hay que aprender a convivir con los síntomas, adaptarse al hospital y a las exigencias de los tratamientos, dar significado a la enfermedad, aceptar el cambio y reorganizarse.

- Fase crónica: es intermedia entre el diagnóstico y la fase terminal. En esta fase hay que compatibilizar las necesidades de la enfermedad con las necesidades de la familia.

- Fase terminal: la sensación de pérdida está presente. Hay que afrontar la muerte y el trabajar el duelo.

La hospitalización, los tratamientos, las secuelas y la muerte del niño son un desafío para la familia ${ }^{4}$.

\section{Hospitalización}

La enfermedad y la hospitalización son las primeras situaciones críticas con las que tienen que enfrentarse los niños y su familia: el carácter extraño del hospital, la separación de la familia y del entorno y el impacto de la enfermedad.

Cuando el niño es hospitalizado se reduce su entorno personal. Su familia, especialmente sus padres, no pueden estar con él siempre que quieran, tampoco sus amigos. Los padres deberán turnarse para hacer compañía al hijo, pudiendo incluso llegar a sentirse mal por no poder estar con él en ciertos momentos en que no les está permitido (exploraciones médicas, tratamientos...), momentos de mayor angustia y ansiedad.

La presencia de los padres así como su participación en los cuidados y otras actividades en la institución hospitalaria resultan vitales para apoyar y mantener las relaciones entre los subsistemas paternal y filial. Del mismo modo, el hecho de estar con el hijo conforta a los propios padres quienes se sienten más útiles por poder ayudar y colaborar en la recuperación.

Pero la hospitalización es una circunstancia "puntual o pasajera». Las altera- 
ciones en el sistema familiar se producen como consecuencia de la aparición de la enfermedad y de sus secuelas.

\section{Tratamientos, recidivas y secuelas}

Los tratamientos de algunas enfermedades pueden provocar efectos desagradables. Esta situación despierta angustia en los padres hasta el punto de preguntarse si merece la pena hacer sufrir al niño. Los efectos adversos les recuerdan que su hijo padece una enfermedad grave, reactivándose así la inquietud por la supervivencia. Esta angustia se agudiza aún más si el niño pide que cese el tratamiento.

Cuando la enfermedad ha remitido, la mayor preocupación de los padres es su reaparición. Aunque en esta etapa mantengan las esperanzas de curación, la ansiedad siempre estará presente en sus vidas.

En el caso de las recidivas toda la familia sufre enormemente. Las reacciones de los padres varían desde renovar las esperanzas de curación hasta una actitud pesimista, pasando por una actitud más pragmática de enfrentarse a la enfermedad. Sienten una mezcla de esperanza y de culpa. La adaptación a las recidivas es más compleja cuanto más largo haya sido el periodo de remisión ${ }^{5}$.

Algunas enfermedades provocan secuelas graves (neurológicas, ortopédicas, urinarias, respiratorias...). Cuando se producen secuelas neurológicas, los padres deben enfrentarse a las alteraciones motoras, sensoriales, cognitivas, emocionales y de comunicación de sus hijos. Estos problemas se convierten en el centro de sus vidas, de sus noches somnolientas, de sus necesidades financieras y de transporte $\mathrm{y}$, en suma, son el desencadenante de los conflictos familiares.

Los padres tienen que reestructurar su vida familiar en función de la enfermedad, pero sin olvidar otros aspectos de su vida, ya que ésta puede ocupar todo su tiempo, absorber toda su atención y todos sus recursos económicos.
La enfermedad es impredecible, puede ser inesperadamente disruptiva, requiere una vigilancia y atención constante y llega a ser una parte intrusa y exigente de la vida familiar, que produce cambios en: las rutinas familiares, las comidas, la asignación de papeles, la división del trabajo y las actividades recreativas o celebraciones festivas, los planes y prioridades de las familias y el modo en que se expresan las emociones $^{6}$.

\section{Muerte}

La muerte de un niño es un tema muy doloroso para los padres, hermanos y profesionales de la salud. La preocupación por cómo tratar al niño moribundo y a su familia es cada vez más evidente en los hospitales.

Los padres, en un comienzo, rechazarán la idea de la muerte o no sabrán lo que se les está diciendo. Pueden reaccionar de forma violenta cuestionando si la enfermedad de su hijo era algo inevitable, con sentimientos de culpa, enojo y desplazamiento de la hostilidad provocada por la enfermedad hacia su cónyuge, hermanos, parientes y personal médico.

Más adelante, aparece la aceptación gradual de la muerte del niño, con sentimientos de tristeza, irritabilidad, intranquilidad y otras reacciones aflictivas típicas. En ocasiones, la culpa de los padres puede intensificarse por el deseo de que la enfermedad acabe, movidos por un sentimiento de descanso y liberación cuando llegan los momentos finales.

La muerte del niño implica la reorganización familiar a largo y corto plazo. La familia tiene que pasar el duelo y esto supone: reconocer la realidad de la pérdida, compartir el dolor (identificar, comprender y dar explicación a los sentimientos, para aceptarlos y encontrar vías para su adecuada canalización), reorganizar el sistema familiar (adaptarse a la nueva vida sin el ser querido) y establecer nuevas relaciones y metas en la vida? 


\section{CARACTERÍSTICAS DE LA ENFERMEDAD}

Las reacciones de las familias dependen de cómo comienza, transcurre y finaliza la enfermedad y de la gravedad de las secuelas ${ }^{8}$.

\section{Comienzo (agudo o gradual)}

Un comienzo agudo conlleva que la familia tenga un tiempo menor de adaptación, que si el comienzo es gradual, y un mayor desgaste de la familia al tener que compatibilizar el mantenimiento de su propia identidad con la atención a las demandas de la enfermedad.

\section{Curso (progresivo o constante)}

Un curso progresivo requiere que la familia tenga que estar en una adaptación continua, por lo que los momentos de respiro son menores.

En las enfermedades de curso constante el enfermo se recupera en mayor o menor medida y la enfermedad se estabiliza, pero siempre queda un déficit o limitación que es asumido por la familia o por el cuidador primario.

Cuando la enfermedad cursa con recaídas, la familia pasa de períodos de remisión de los síntomas, durante los cuales desarrolla sus rutinas, a periodos de exacerbación de los mismos, viviendo bajo el estrés de la posibilidad de una nueva recaída.

\section{Resultado de la enfermedad}

En las enfermedades que amenazan la existencia se puede producir un duelo anticipado tanto en el enfermo como en la familia, aislando al enfermo y apartándolo de ciertas tareas o actividades. Asimismo, la incertidumbre de la muerte puede provocar la sobreprotección.

\section{Discapacidad}

El grado de discapacidad implica ajustes en la adaptación relacionados con el comienzo, curso y resultado de la enfermedad. Si ésta es progresiva, la discapacidad se produce en las últimas fases de la enfermedad, lo que permite a la familia anticipar recursos y estrategias.

\section{ALTERACIONES ESTRUCTURALES, PROCESUALES Y EMOCIONALES}

Las alteraciones más comunes son las estructurales, procesuales y emocionales ${ }^{9}$.

\section{Alteraciones estructurales}

Hacen referencia a los cambios en los roles y funciones de los miembros de la familia, a la adscripción del rol de "cuidador primario", a la rigidez en la interacción familiar, en la relación familia/persona afectada y en las interacciones de la familia con los servicios asistenciales y el aislamiento social.

\section{Cambios en los roles, funciones y jerarquía familiar}

Los cambios en los roles y funciones sean posiblemente los más profundos y complejos a los que se enfrenta la familia como consecuencia de una enfermedad crónica, pues se ve obligada a negociar roles y funciones que hasta el momento desempeñaba el miembro enfermo. Cuando el que enferma es uno de los hijos el impacto estructural es menor, ya que el niño no tiene muchas responsabilidades familiares, pero el impacto emocional puede llegar a ser muy profundo.

Del mismo modo que la familia tiene que adaptarse a la enfermedad, debe hacerlo a la vida cotidiana; en algunas ocasiones, nos encontramos con familias de pacientes crónicos gravemente discapacitados que parecen cooperar durante la hospitalización, pero luego plantean graves dificultades en el alta.

Si quien enferma es un niño pequeño, el deseo de desarrollar plenamente su actividad, que ha estado limitada durante su 
hospitalización, puede crear situaciones incómodas generadas por los propios padres, que, incapaces de controlarlo, insisten en que respete normas que el niño se niega a aceptar.

Si es un adolescente, el proceso de emancipación puede quedar cuestionado. En casa se le somete a una disciplina que ya no corresponde a su edad, lo que genera conflictos al tener que negociar la autonomía ya conseguida en etapas anteriores. En estos casos, la orientación a los padres ha de dirigirse al reconocimiento del esfuerzo de su hijo por superarse y a la asignación de responsabilidades acordes a su capacidad y posibilidad de asunción.

\section{Adscripción del rol de "cuidador primario»}

El «cuidador primario" es quien asume el cuidado de la persona enferma. Habitualmente, la adscripción sigue criterios socioculturales que designan a las mujeres; en el caso de los niños, suelen ser las madres. Es importante que el cuidador primario tenga períodos de respiro para evitar su desgaste, ya que la calidad del cuidado dependerá en gran medida de que el cuidador descanse.

Así, una vez diagnosticada una enfermedad, debe identificarse la persona que asumirá la atención del enfermo. Hay que adiestrarle y ayudarle para que ataje las situaciones de crisis que le puede acarrear su función ${ }^{10}$.

\section{Rigidez en la interacción familiar}

También puede originarse una rigidez en la interacción familiar y en la relación familia-persona afectada debido a las dificultades de la familia para cambiar su organización, especialmente, al cambiar de fase.

Las coaliciones y las exclusiones emocionales caben entenderse en términos de rigidez: el cuidador primario y el enfermo desarrollan una relación estrecha de la que los restantes miembros quedan excluidos. En ocasiones esa relación privilegiada es resentida por algún otro familiar, como es el caso de los hermanos.

También aparece la sobreprotección como respuesta en la que se exime al enfermo de realizar cualquier tipo de esfuerzo, limitando su propia vida, lo que provoca muchos conflictos durante el forcejeo de lo que puede y no puede hacer. El niño sobreprotegido puede darse cuenta de que ocupa un lugar privilegiado en la familia, lo que confirma sus sentimientos de fragilidad y ansiedad respecto a su supervivencia. Los padres podrían tener problemas para hablar con su hijo sobre la enfermedad y los tratamientos.

\section{Rigidez entre las familias y servicios asistenciales}

La rigidez también es común en las interacciones entre la familia y los servicios asistenciales. Los profesionales pueden enviar mensajes negativos a la familia restándole competencia, por lo que la familia suele delegar sus funciones en ellos. Frecuentemente, los padres llegan a hacerse expertos de la enfermedad de su hijo y pueden llegar a competir con el personal sanitario.

Estos conflictos entre el personal y la familia son comprensibles si se tienen en cuenta las tensiones bajo las cuales trabaja el equipo médico-sanitario y las angustias de los padres que tienen hijos enfermos. Los padres no pueden entender su situación de forma tan objetiva y racional como el personal sanitario y tienen sentimientos que para los profesionales son incongruentes, llegando en ocasiones a rivalizar con ellos si consideran que la relación con el niño peligra.

Otras veces, el desconocimiento de los padres sobre la enfermedad puede originar desconfianza hacia el personal. Es importante ofrecer información clara y esperanzadora en todas las etapas de la enfermedad. 


\section{Aislamiento social}

Otra característica de muchas de las familias afectadas es el aislamiento que se produce al reducir los tiempos de compartir con los amigos, los dedicados al ocio, al trabajo... Este aislamiento puede estar relacionado con el rechazo o el estigma de la enfermedad.

\section{Alteraciones procesuales}

La enfermedad fuerza un momento centrípeto de transición en la familia, de carácter para adaptarse a las necesidades que aquélla impone. Puede entenderse el ciclo familiar como la alternancia de momentos centrípetos y centrífugos. Los momentos centrípetos son aquéllos en los que la familia tiene que realizar tareas que son internas a la familia (se mantiene la estructura familiar); y por momentos centrífugos los que, por el contrario, implican tareas externas a la familia (se generan nuevas estructuras familiares). En general, la aparición de una enfermedad fuerza un momento centrípeto. Si la enfermedad coincide con un momento centrífugo, puede sacar a la familia de su momento natural. Si coincide con un momento centrípeto, éste se prolongará.

\section{Alteraciones emocionales}

Las emociones de la familia son diferentes en cada etapa de la enfermedad y suelen ser muy complejas.

\section{Emociones según las etapas de la enfermedad}

La familia que se enfrenta a la experiencia de una enfermedad responde de manera diferente en función de las fases de la enfermedad. Ante el diagnóstico la reacción es de shock y pánico. La primera fase de desintegración cursa con depresión, cólera y culpa. En la fase de ajuste, la familia busca información que le anime y se va acomodando emocionalmente; también debe enfrentarse a la sociedad (dar explicaciones...). Durante la fase de reintegración, la familia trata de poner la enfermedad en su lugar y de integrarla en la vida familiar.

\section{Respuestas emocionales complejas}

La presencia de un hijo con una enfermedad constituye un factor potencial que puede llegar a perturbar el funcionamiento familiar y ese funcionamiento determinará la reacción del niño ante la enfermedad. Los padres responderán no sólo en función de la enfermedad, del tratamiento y de la forma como reaccione el niño, sino también según sus propios sentimientos y problemas personales, lo que significa que tanto las reacciones del niño enfermo como la de sus padres son interdependientes.

La familia afectada por una enfermedad responde emocionalmente de manera compleja: se entremezclan afectos positivos y negativos. Sus miembros pueden experimentar sentimientos de pena, impotencia, agresividad, culpabilidad, ambivalencia (deseos de ayudar al enfermo y, a su vez, sentirlo como una carga o desear que muera para que no sufra), injusticia, temor al futuro... Como respuesta, los familiares pueden sufrir un derrumbe psicológico (depresión, ansiedad...) o físico (el cuidador primario puede enfermar), o abandono del enfermo.

Si el niño tiene hermanos, éstos también reaccionarán ante el desequilibrio de la familia. Los hermanos igualmente experimentan sentimientos de culpa (por alegrarse de no ser ellos los enfermos y por tener sentimientos negativos hacia su hermano si los padres propician a éste más atención). Otras veces podrán sentirse desplazados si entienden las atenciones al niño enfermo como favoritismo, deseando también estar enfermos. Los padres, a su vez, pueden llegar a reducir la atención hacia los demás hijos, olvidando sus necesidades o esperando de ellos lo que no podrá conseguir el niño enfermo.

La enfermedad del hermano puede producirles un impacto tan fuerte que les lleve a desarrollar trastornos del comportamiento, fracaso escolar o incluso sufrir ellos mismos síntomas físicos: dolor de cabeza, de estómago o síntomas parecidos al hermano enfermo que, en ocasiones, requieran consulta médica. Los hermanos con capacidad de entender la enfermedad pueden preocuparse 
al ver en sus iguales la afección y se imaginan que ellos mismos pueden enfermar.

Las experiencias negativas con los sistemas de salud suelen dificultar la confianza en los profesionales y la colaboración con los mismos. También los sentimientos hacia los profesionales están condicionados por los valores culturales ${ }^{11}$. Por ejemplo, según las culturas, los padres respetan a los médicos y saben expresar sus sentimientos y recibir su ayuda o exageran los problemas para que los profesionales se den cuenta de lo dura que es su situación.

El sentido que la familia dé a la enfermedad y el grado de control que ejerza sobre la misma condicionará el ajuste emocional a la situación: depende de su sistema de creencias y de la cultura en la que está inmersa. Ligado al control de la enfermedad está la posibilidad de mantener la esperanza, cierta calidad de vida y cierta dignidad.

La conspiración de silencio en torno a la enfermedad del paciente o el temor a la transmisión genética pueden ser otro tipo de alteraciones en la respuesta emocional.

\section{APOYO Y COLABORACIÓN CON LA FAMILIA}

En las últimas décadas estamos asistiendo a un cambio de enfoque en el tratamiento de las enfermedades crónicas pediátricas: ya no son consideradas como un problema médico, sino también como un problema familiar y social ${ }^{12,13}$.

Desde ese punto de vista, la familia cobra una gran relevancia y los profesionales deben colaborar con ésta y apoyar$\mathrm{la}^{14}$. Por ello es imprescindible: respetar la singularidad de la familia, basarse en sus necesidades, tener una comprensión multidimensional del problema, desarrollar competencias en los padres y la resiliencia, contar con los sistemas de apoyo del entorno natural, y coordinar la acción de los servicios públicos (sanitarios, educativos y de asistencia social) con los que ofrece la sociedad civil (asociaciones, instituciones privadas...).

\section{Singularidad de las familias}

Las respuestas de la familia a la enfermedad no suelen ser patológicas, sino que se derivan de las expectativas, experiencias y condicionamientos culturales de la familia hacia la enfermedad.

Cada familia reacciona y vive la enfermedad de manera diferente y singular. Todas las familias pasan por las diferentes fases de adaptación (negación, ira, pacto, depresión y aceptación) pero sus reacciones y resoluciones son diferentes. La familia desarrolla sus propias estrategias como una respuesta normal a un sistema desequilibrado provocado por la enfermedad.

Es la familia la que asume la responsabilidad del manejo del día a día del enfermo crónico y es la que mejor nos puede enseñar acerca del impacto de la enfermedad crónica sobre su vida y de aquellas actitudes y conductas que son más útiles para afrontar satisfactoriamente el problema.

Desde este punto de vista, los profesionales, más que intervenir, deben acompañar y ayudar a las familias a buscar significado a un sistema desequilibrado porque éstas tienen experiencias equivalentes a la de los profesionales en el tratamiento de la enfermedad ${ }^{15}$.

\section{Comprensión multidimensional}

Las reacciones de la familia dependen de numerosos factores como experiencia en situaciones de crisis y problemas médicos, estatus socio-económico, cultura, nivel de conocimientos, calidad de los servicios sanitarios y sistemas de apoyo. Cada familia es una unidad diferenciada de las otras, con antecedentes socio-culturales, experiencias y recursos propios.

Este enfoque multidimensional nos permite comprender el sentido que cada familia le otorga a la vida antes y después de la enfermedad. La evaluación de su problemática debe centrarse en cómo sus miembros juzgan los acontecimientos estresantes y en qué estrategias han utilizado para superar los problemas. Es más importante evaluar las prioridades y necesidades de las 
familias que realizar evaluaciones clínicas. Las familias necesitan ser comprendidas y no interpretadas. Sus valores culturales deben ser respetados y no cuestionados.

Asimismo, la intervención debe ser acorde con los objetivos de cada familia. Sus aspiraciones después de la enfermedad son las mismas que las de todas las familias y su interpretación depende de cada una.

La familia debe mantener su identidad frente la enfermedad y los profesionales deben estar preparados para entender sus puntos de vista.

\section{Desarrollar competencias: resiliencia}

Este enfoque multidimensional de la crisis nos explica el porqué algunos niños y sus familias son más resistentes (resiliencia) o más vulnerables ante la enfermedad y sus secuelas. Una familia con resiliencia es capaz de movilizar sus recursos, utilizar los sistemas de apoyo y poner sus emociones y circunstancias bajo control. Las familias más vulnerables están inmovilizadas y no controlan la situación.

La resiliencia es el conjunto de procesos sociales y personales que posibilitan conseguir el bienestar físico y psicológico a pesar de las adversidades. Es la capacidad que tienen determinados sujetos y familias de sobreponerse a las adversidades, de auto-regenerarse de determinadas heridas o traumas y de sobreponerse a acontecimientos desestabilizadores ${ }^{16-19}$.

En el caso de una enfermedad crónica pediátrica, las familias con resiliencia son capaces de adaptarse dentro y fuera del hospital, disponen de apoyos sociales, se dotan de recursos económicos y sociales y mantienen una comunicación abierta en la familia y con el personal del hospital. Conciben la enfermedad como una oportunidad de superación, encuentran sentido al sufrimiento, buscan activamente estrategias de afrontamiento positivas, valoran el hecho de que su hijo esté superando la enfermedad y reorganizan prioridades y roles.

En 1998 Froma Walsh elaboró el concepto de "resiliencia familiar", entendiéndola como los procesos interactivos que fortalecen al individuo y a la familia en el transcurso del tiempo ${ }^{20}$. Estos procesos interactivos son: reconocer los problemas y las limitaciones, hablar abierta y claramente acerca de ellos, analizar los recursos personales y familiares existentes y organizar y reorganizar las estrategias tantas veces como sea necesario, revisando y evaluando los logros y las pérdidas. Los factores que determinan la capacidad de resiliencia de la familia son: de desarrollo individual e interpersonal, estratégicos y de visión (Tabla 1).

Tabla 1. Factores que determinan la capacidad de resiliencia de la familia.

\begin{tabular}{|c|l|l|c|}
\hline Desarrollo individual & Desarrollo interpersonal & \multicolumn{1}{|c|}{ Estratégicos } & De visión \\
\hline Capacidad de desarrollar & Capacidad para & Capacidad para fijar & Capacidad para \\
en cada miembro: & desarrollar la empatía & metas & desarrollar: \\
- Su identidad y rol & - El apoyo mutuo & - Valores, propósitos y \\
- Flexibilidad & - La comunicación & - Reflexionar & expectativas \\
perceptual y & - El sentido del humor & - Resolver conflictos & - Optimismo y fe \\
conductual & - Introspección & - Pedir apoyos y & \\
- Autoestima y & individual y grupal & recursos & \\
confianza & & - Evaluar resultados & \\
- Competencia & & & \\
- Capacidad de & & & \\
distanciarse de los & & & \\
mensajes negativos & & & \\
\hline
\end{tabular}




\section{Servicios centrados en el consumidor y colaborativos}

Los servicios deben organizarse en función de las necesidades de los usuarios (enfermos y familias) y no de los profesionales $^{21,22}$. Las necesidades de una familia pueden concretarse en:

- Las que tienen un impacto en la merma de la calidad de vida de sus miembros.

- Las que tienen relación con el papel que desempeña la familia en la mejora de la calidad de vida del niño enfermo.

En el primer supuesto la familia es receptora de servicios y en el segundo, la familia es proveedora. La familia, cuando es receptora, puede participar en servicios de respiro familiar, de orientación, de apoyo (grupos de autoayuda de padres y familiares), de terapia familiar y de redes sociales (asociaciones).

Cuando la familia es proveedora de servicios participa en el tratamiento del hijo (hospitalización domiciliaria...) y en las actividades formativas. Las familias deben compartir responsabilidades con los profesionales y ser parte activa en la toma de decisiones y en su implementación, ya que el control de la enfermedad permite que el niño tenga una mejor calidad de vida: evita los reingresos hospitalarios, normaliza las relaciones sociales y favorece la integración escolar y social.

Se debe conseguir que la familia sea capaz de gestionar y dirigir los cuidados necesarios al niño enfermo, apoyándose en los recursos disponibles en su entorno social (voluntariado, asociaciones de afectados, de ocio, vecinales...). Hay que ser creativos y medir el coste/eficacia de los programas de entrenamiento.

Los comités clínicos deben trabajar en colaboración con los servicios de la comunidad (educativos, servicios sociales, deportivos...). Asimismo, se necesitan investigadores multidisciplinares para evaluar los efectos de los diferentes tipos de intervención y de programas.
La coordinación debe entenderse como un proceso colaborativo de análisis de los servicios disponibles, de planificación, de puesta en práctica, de coordinación y de evaluación de la atención proporcionada al niño enfermo y no como la mera organización del uso de los servicios asistenciales.

\section{CONCLUSIONES}

Colaborar y ayudar a las familias con un hijo enfermo es una tarea importante para los profesionales que en distintos ámbitos trabajan con el niño enfermo. La colaboración de la familia es fundamental para aumentar la calidad de vida del niño y favorecer su integración social y escolar.

Es imprescindible que los profesionales conozcan cómo afecta la enfermedad a las familia, que sean receptivos a sus preocupaciones y necesidades y que favorezcan el desarrollo de estrategias de afrontamiento activas que permitan a los padres solucionar los problemas, buscar información válida y apoyo profesional, reestructurar sus valores, controlar sus emociones y buscar apoyo emocional en su familia y amigos ${ }^{23}$, es decir, que logren la resiliencia. Así, los padres competentes ${ }^{24}$ :

- Saben manejar adecuadamente la enfermedad y proporcionan los cuidados que necesitan los hijos.

- Participan activamente en el tratamiento que se les administra en el hospital y son candidatos a la hospitalización domiciliaria.

- Buscan información adecuada y se la proporcionan a sus hijos.

- Ayudan a sus hijos a desarrollar destrezas para el manejo de la enfermedad.

- Controlan los sentimientos negativos y la ansiedad, y tienen una actitud positiva respecto a la resolución de los problemas, evitando caer en la depresión. Del mismo modo, ayudan a los hijos a controlar sus emociones y saben manejar las emociones negativas de los profesionales. 
- Aceptan las dificultades de los hijos, tienen más recursos para solucionar los problemas y colaboran estrechamente con los profesionales de los diferentes servicios: sanitarios, educativos y de asistencia social.

En última instancia, la colaboración y ayuda a la familia tiene repercusiones positivas en la familia y en los servicios públicos. En la familia un mejor rendimiento escolar, personal y social del niño enfermo y de sus hermanos y un mayor bienestar personal, emocional y profesional de los padres, con la consiguiente mejora de la calidad de vida de la familia en su conjunto. En los sistemas públicos de salud, educación y servicios sociales: se utilizan menos servicios especializados o extraordinarios, lo que constituye un ahorro en el gasto público.

\section{BIBLIOGRAFÍA}

1. YlVISAKER M. Traumatic brain injury in children and adolescents: Introduction. En: Ylvisaker M. Traumatic brain injury rehabilitation. Washigton: Buttrworth- Heinemann, 1998: 1-10.

2. FIOREnTINo MT. La construcción de la resiliencia en el mejoramiento de la calidad de vida y la salud. Suma Psicológica 2008; 15: 95-114.

3. WAALAND PK. Families of Children with Traumatic Brain Injury. En M Ylvisaker Traumatic Brain Injury Rehabilitation. Washigton, Buttrworth-Heinemann, 1998: 345-368.

4. Grau C. Atención educativa al alumnado con enfermedades crónicas o de larga duración. Málaga: Aljibe, 2004.

5. Grau C. Impacto psicosocial del cáncer infantil en la familia. Educación, Desarrollo y Diversidad 2002; 5: 67-87.

6. González S, Steinglass, Reiss D. Intervenciones centradas en familias con miembros que tienen dolencias crónicas. George Washintong University, 1987.

7. WALSH F, McGolDRICKC M. La pérdida y el ciclo vital de la familia. En Jaes Falicov (comp.). Transiciones de la familia. Continuidad y cambio en el ciclo de la vida. Buenos Aires: Amorrortu, 1991: 429-464.

8. Rolland JS. Familia, enfermedad y crisis. México: Gedisa, 2000.

9. NAVARRo J. Familia y discapacidad. Manual de intervención psicosocial. Junta de Castilla y León. Consejería de Sanidad y Bienestar Social y Real Patronato de prevención y de atención a personas con minusvalía, 1999.

10. Chacón M, Grau J. La familia como dadora de cuidados. En: M Gómez. Cuidados Paliativos. Atención integral a enfermos terminales (II). Canarias: I.C.E.P.S.S. (Instituto Canario de Estudios y Promoción Social y Sanitaria) 1998: 975-986.

11. Álunarez F, Fortin S, BibEAu G. La práctica pediátrica en un medio cultural plural: una experiencia en curso. Arch Argent Pediatr 2008; 106: $236-241$.

12. González-Lamuño D, García Fuentes M. Enfermedades raras en pediatría. An Sist Sanit Navar 2008; 31 (Supl. 2): 21-29.

13. YuRSS I. Atención a la familia: otra forma de enfocar los problemas de salud en atención primaria. Instrumentos de abordaje familiar. An Sist Sanit Navar 2001; 24 (Supl. 2): 73-82.

14. Fuertes MC, MaYA MU. Atención a la familia: la atención familiar en situaciones concretas. An Sist Sanit Navar 2001; 24 (Supl. 2): 83-91.

15. ÁlLAREz LY. Modelos explicativos y de intervención psicosocial de la enfermedad crónica. MedUNAB 2006, 9: 250-256.

16. Cyrulnik B. Los patitos feos. La resilencia: una infancia infeliz no determina la vida. Barcelona: Gedisa 2002.

17. Cyrulnick B. La maravilla del dolor. El sentido de la resiliencia. Barcelona: Ed. Granica 2001.

18. ManciauX M. La resiliencia: resistir y rehacerse. Barcelona: Gedisa 2003.

19. KeRn E, Moreno-Jiménez B. Resiliencia de niños enfermos crónicos: aspectos teóricos. Psicología en estudio 2007; 12: 81-86.

20. WALSH F. El concepto de resiliencia familiar: crisis y desafío. Sistemas Familiares 1998; 14: 11.

21. González P. Experiencias y necesidades percibidas por los niños y adolescentes con cáncer y sus familias. Nur Inv 2005; 16: 1-15.

22. Castillo E, Chesla CA. Viviendo el cáncer de un (a) hijo (a). Colomb Med 2003; 34: 155163.

23. De la Huerta R, Corona J, Méndez J. Evaluación de los estilos de afrontamiento en cuidadores primarios de niños con cáncer. Neurol Neurocir Psiquiat 2006, 39: 46-51.

24. EsPADA MC. Intervención psicoeducativa para el desarrollo de habilidades de afrontamiento en padres de hijos con cáncer. Tesis Doctoral. Valencia: Universidad de Valencia, 2009. 